University of Wollongong

Research Online

\title{
Olanzapine increases AMPK-NPY orexigenic signaling by disrupting H1R-GHSR1a interaction in the hypothalamic neurons of mice
}

\author{
Xiaoqi Chen \\ University of Wollongong \\ Yinghua $\mathrm{Yu}$ \\ University of Wollongong, yinghua@uow.edu.au \\ Peng Zheng \\ University of Wollongong, pz633@uowmail.edu.au \\ Tiantian Jin \\ University of Wollongong \\ Meng $\mathrm{He}$
}

See next page for additional authors

Follow this and additional works at: https://ro.uow.edu.au/ihmri

Part of the Medicine and Health Sciences Commons

\section{Recommended Citation}

Chen, Xiaoqi; Yu, Yinghua; Zheng, Peng; Jin, Tiantian; He, Meng; Zheng, Mingxuan; Song, Xueqin; Jones, Alison L.; and Huang, Xu-Feng, "Olanzapine increases AMPK-NPY orexigenic signaling by disrupting H1RGHSR1a interaction in the hypothalamic neurons of mice" (2020). Illawarra Health and Medical Research Institute. 1511.

https://ro.uow.edu.au/ihmri/1511

Research Online is the open access institutional repository for the University of Wollongong. For further information contact the UOW Library: research-pubs@uow.edu.au 


\title{
Olanzapine increases AMPK-NPY orexigenic signaling by disrupting H1R-GHSR1a interaction in the hypothalamic neurons of mice
}

\author{
Abstract \\ Second generation antipsychotics, particularly olanzapine, induce severe obesity, which is associated with \\ their antagonistic effect on the histamine $\mathrm{H} 1$ receptor $(\mathrm{H} 1 \mathrm{R})$. We have previously demonstrated that oral \\ administration of olanzapine increases the concentration of neuropeptide Y (NPY) in the hypothalamus of \\ rats, accompanied by hyperphagia and weight gain. However, it is unclear if the increased NPY after \\ olanzapine administration is due to its direct effect on hypothalamic neurons and its H1R antagonistic \\ property. In the present study, we showed that with an inverted U-shape dose-response curve, olanzapine \\ increased NPY expression in the NPY-GFP hypothalamic neurons; however, this was not the case in the \\ hypothalamic neurons of H1R knockout mice. Olanzapine inhibited the interaction of H1R and GHSR1a \\ (ghrelin receptor) in the primary mouse hypothalamic neurons and NPY-GFP neurons examined by \\ confocal fluorescence resonance energy transfer (FRET) technology. Furthermore, an H1R agonist, FMPH \\ inhibited olanzapine activation of GHSR1a downstream signaling PAMPK and transcription factors of NPY \\ (pFOXO1 and pCREB) in the hypothalamic NPY-GFP cell. However, an olanzapine analogue (E-Olan) with \\ lower affinity to H1R presented negligible enhancement of pCREB within the nucleus of NPY neurons. \\ These findings suggest that the H1R antagonist property of olanzapine inhibits the interaction of H1R and \\ GHSR1a, activates GHSR1a downstream signaling pAMPK-FOX01/pCREB and increases hypothalamic \\ NPY: this could be one of the important molecular mechanisms of H1R antagonism of olanzapine- \\ induced obesity in antipsychotic management of psychiatric disorders.

\section{Disciplines} \\ Medicine and Health Sciences

\section{Publication Details} \\ Chen, X., Yu, Y., Zheng, P., Jin, T., He, M., Zheng, M., Song, X., Jones, A. \& Huang, X. (2020). Olanzapine \\ increases AMPK-NPY orexigenic signaling by disrupting H1R-GHSR1a interaction in the hypothalamic \\ neurons of mice. Psychoneuroendocrinology, 114 104594-1-104594-10.
}

\section{Authors}

Xiaoqi Chen, Yinghua Yu, Peng Zheng, Tiantian Jin, Meng He, Mingxuan Zheng, Xueqin Song, Alison L. Jones, and Xu-Feng Huang 


\title{
Olanzapine increases AMPK-NPY orexigenic signaling by disrupting H1R-GHSR1a interaction in the hypothalamus
}

Xiaoqi Chen ${ }^{1,2}$, Yinghua $\mathrm{Yu}^{* 3}$, Peng Zheng 2 , Tiantian $\mathrm{Jin}^{2}$, Meng $\mathrm{He}^{4}$, Mingxuan Zheng ${ }^{3}$, Xueqin Song $^{5}$, Alison Jones ${ }^{2}$, Xu-Feng Huang ${ }^{2 *}$

\begin{abstract}
Affiliations:
1. Department of Endocrinology and Rheumatology, Zhongnan Hospital of Wuhan University, Wuhan 430071, China

2. Illawarra Health and Medical Research Institute and School of Medicine, University of Wollongong, NSW2522, Australia
\end{abstract}

3. Department of Pathogen Biology and Immunology, Xuzhou Medical University and Jiangsu Key Laboratory of Immunity and Metabolism, Xuzhou, Jiangsu 221004, China

4. School of Chemistry, Wuhan University of Technology, Wuhan, China

5. School of Psychiatry, The first affiliated hospital of Zhengzhou University, Zhengzhou, China

*Corresponding author:

Professor Xu-Feng Huang, MD, PhD, DSc, School of Medicine, University of Wollongong, Northfields Avenue, NSW2522, Australia. E-mail: xhuang@uow.edu.au

Professor Yinghua Yu, MD, PhD, Department of Pathogen Biology and Immunology, Xuzhou Medical University and Jiangsu Key Laboratory of Immunity and Metabolism, Xuzhou, Jiangsu 221004, China. E-mail: yinghuahillary@foxmail.com 


\begin{abstract}
Second generation antipsychotics, particularly olanzapine, induce severe obesity, which is associated with their antagonistic effect on the histamine $\mathrm{H} 1$ receptor (H1R). We have previously demonstrated that oral administration of olanzapine increases the concentration of neuropeptide $\mathrm{Y}$ (NPY) in the hypothalamus of rats, accompanied by hyperphagia and weight gain. However, it is unclear if the increased NPY after olanzapine administration is due to its direct effect on hypothalamic neurons and its H1R antagonistic property. In the present study, we showed that with an inverted U-shape dose-response curve, olanzapine increased NPY expression in the NPYGFP hypothalamic neurons; however this was not the case in the hypothalamic neurons of H1R knockout mice. Olanzapine inhibited the interaction of H1R to GHSR1a (ghrelin receptor) in the primary mouse hypothalamic neurons and NPY-GFP neurons examined by confocal fluorescence resonance energy transfer (FRET) technology. Furthermore, an H1R agonist, FMPH inhibited olanzapine activation of GHSR1a downstream signaling pAMPK-UCP2 and transcription factors of NPY (pFOXO1 and pCREB) in the hypothalamic NPY-GFP cell. However, an olanzapine analogue (OlzEt) with lower affinity to H1R presented negligible enhancement of pCREB within the nucleus of NPY neurons. These findings suggest that the H1R antagonist property of olanzapine inhibits the interaction of H1R to GHSR1a, activates GHSR1a downstream signaling pAMPK-UCP2-FOXO1/pCREB and increases hypothalamic NPY, which could be one of important molecular mechanism of H1R antagonism of olanzapine-induced obesity in antipsychotic management of psychiatric disorders.
\end{abstract}

Key words: antipsychotic drug, histamine H1 receptor, neuropeptide Y, hypothalamus, ghrelin receptor 1a, weight gain, BMI 


\section{Introduction}

Second generation antipsychotic drugs (SGAs) are widely prescribed for a growing number of mental illnesses including schizophrenia, bipolar disorder, dementia, major depression, autism, and Tourette's disease [1]. SGAs can cause serious side effects including body weight gain, obesity and metabolic syndrome, leading to further chronic life-threatening diseases and medication noncompliance [2;3]. Olanzapine has one of the highest obesogenic liabilities of all SGAs [4;5], with a significant body of evidence demonstrating its association with metabolic hormone imbalance, hyperphagia, and adiposity $[6 ; 7 ; 8]$. Despite a vast body of literature examining antipsychotic metabolic side-effects, the molecular mechanism underlying SGA-induced obesity is still largely unclear.

Neuropeptide Y (NPY) is an orexigenic neuropeptide in the hypothalamus and plays an important role in regulating positive energy balance. Previously, we found that oral administration of olanzapine increased NPY level in the hypothalamus of rats with hyperphagia and weight gain [9]. However, it is unclear if the activation of NPY induced by olanzapine is due to its direct effect on hypothalamic neurons or a secondary effect of obesity. Therefore, the precise mechanism of olanzapine induced NPY should be investigated at cellular level. In the hypothalamic arcuate nucleus (Arc), more than 90\% NPY neurons express the growth hormone secretagogue receptor 1a (GHSR1a; also called the 'ghrelin receptor') [10]. GHSR1a promotes mitochondrial $\beta$ oxidation through AMP-activated protein kinase (AMPK) phosphorylation and subsequent activity of carnitine palmitoyl transferase 1 (CPT1), which transports fatty acids into the mitochondria, and uncoupling protein-2 (UCP2) [11; 12]. GHSR1a-induced activation of the 
AMPK-CPT1-UCP2 pathway increases NPY mRNA expression by modulating intracellular transcriptional factors, forkhead box $\mathrm{O} 1$ (FOXO1) and the phosphorylated cAMP-response element-binding protein (pCREB), which are translocated to the nucleus to initiate NPY promoter activity $[13 ; 14 ; 15 ; 16]$. Therefore, GHSR1a-AMPK- FOXO1/p-CREB play important role in activation of NPY in the hypothalamus.

Olanzapine's obesogenic effect is linked to its antagonistic effect on the histamine $\mathrm{H} 1$ receptors. It is reported that H1R antagonism of the antipsychotics activates hypothalamic AMP-kinase to increase food intake and induce weight gain $[17 ; 18 ; 19]$. H1R mRNA is expressed in the Arc [20] where GHSR1a is highly expressed. Accumulating data over the last few years have shown that the GHS-R1a can form a dimer with G-protein coupled receptors involved in appetite signaling and reward, including the GHS-R1b, the melanocortin 3 (MC3), dopamine (D1 and D2), and serotonin 2C (5-HT2C) receptors [21]. Both H1R and GHSR1a regulate AMPK and are colocalized in the hypothalamic neurons, H1R and GHSR1a may interact and regulate AMPK signaling in the hypothalamus, responsible for olanzapine's H1R antagonism effect on activation of AMPK and its downstream NPY for positive energy balance.

In the present study, we examined the H1R antagonism of olanzapine in regulation of NPY in the hypothalamic neurons by using cultured primary hypothalamic neurons, NPY-GFP cell line and hypothalamic A59-NPY neurons. Furthermore, interaction of H1R and GHSR1a in hypothalamic neurons was investigated by confocal fluorescence resonance energy transfer (FRET) technology, as well as the effects of olanzapine on the interaction and GHSR1a signaling pathways (AMPKFOXO1/p-CREB) were also examined. 


\section{Results}

\section{Olanzapine increased NPY expression in NPY-GFP cell line associated with H1R property.}

NPY is one of the most important neurotransmitters stimulating positive energy balance and promoting weight gain [22]. We have previously shown that olanzapine increases hypothalamic NPY of rats with hyperphagia and weight gain [23]. To further testify whether or not olanzapine can directly increase NPY in the hypothalamic neurons, we measured the dosage effects of olanzapine in NPY-GFP cells. We observed an inverted U-shape dose-response effect, where olanzapine increased NPY (Fig 1A). Olanzapine at 10 and $25 \mu \mathrm{M}$ increased NPY concentrations with the maximum effect at $25 \mu \mathrm{M}$, while olanzapine at 1,50 and $100 \mu \mathrm{M}$ did not significantly affect NPY concentrations (Fig 1A). Olanzapine's H1R antagonist property has been reported to increase NPY in an in vivo study [18]. Here, we examined the effects of pyrilamine (a selective antagonist of H1R) and FMPH (a selective agonist of H1R) on NPY in NPY-GFP cells. Pyrilamine increased NPY at 1, 5, and $10 \mu \mathrm{M}$ (Fig 1B), while FMPH (from 0.5-5 $\mu \mathrm{M}$ ) decreased NPY concentrations (Fig 1C). These data demonstrated that both olanzapine and the H1R antagonist increased NPY, but the H1R agonist reduced NPY concentrations in hypothalamic neurons.

\section{Olanzapine does not increase hypothalamic NPY of H1R knockout heterozygous mice.}

To further investigate the H1R of olanzapine regulating hypothalamic NPY, immunocytochemistry-based co-localization of endogenous NPY and H1R was applied in cultured neonatal hypothalamus neurons of H1R knockout mouse in response to olanzapine. In cultured primary hypothalamic neurons, hypothalamic neurons from H1R knockout mouse showed significantly lower H1R levels compared with wildtype mice (Fig 2A and 2B). Olanzapine significantly increased NPY concentrations in wildtype mice $(\mathrm{P}<0.01)$, but not in $\mathrm{H} 1 \mathrm{R}$ knockout 
mice (Fig $2 \mathrm{~A}$ and $2 \mathrm{C}$ ). The results suggest that H1R is mechanistically essential for olanzapine increasing NPY in the hypothalamic neurons.

\section{Olanzapine inhibited the interaction of H1R to GHSR1a in the hypothalamic neurons.}

Hypothalamic NPY neurons express both H1R and GHSR1a [24; 25]. GHSR1a is constantly active, even in an absence of a natural ligand ghrelin. GHSR1a dimerizes with a wide array of receptors including D2R. Since both H1R and GHSR1a regulate NPY, we examined if there is interaction between H1R and GHSR1a and if olanzapine can regulate their interaction. First, immunocytochemistry revealed that mouse postnatal hypothalamic Arc neurons (DIV 10) endogenously expressed H1R and GHSR1a, which co-localized in NPY neurons (Fig. 3A). Confocal FRET displayed the heterodimers of H1R and GHSR1a in NPY neurons (Fig 3B). The control group showed that no FRET was detected without either H1R antibody (acceptor, Fig 3C) or GHSR1a antibody (donor, Fig 3D) applied in these hypothalamic NPY neurons. Therefore, our results demonstrated a physical interaction and receptor heterodimer complex formation between the natively expressed H1R and GHSR1a in hypothalamic NPY neurons. FRET efficiencies (FRETeff) for the sensitized emission (SE) in response to olanzapine, FMPH, and their combination were measured. In some neurons, FMPH $(5 \mu \mathrm{M})$ significantly increased FRET efficiency $(\mathrm{p}<0.05)$, while olanzapine $(25 \mu \mathrm{M})$ and PYR $(5 \mu \mathrm{M})$ decreased FRET efficiency $(\mathrm{p}<0.01$ Fig 3E). Similarly, in the dendrite of neurons, FRET efficiency was significantly increased by FMPH, and decreased by olanzapine and PYR (Fig 3F).

Next, we further confirmed that H1R and GHSR1a were co-localized in NPY-GFP cell line (Fig 4A). Confocal FRET showed that H1R and GHSR1a were able to form heterodimers in the NPYGFP cell (Fig 4B). A combination of FMPH and olanzapine decreased FRET efficiency compared 
with FMPH alone ( $<<0.01$, Fig 4C), suggesting that H1R antagonism of olanzapine blocks H1R agonist effect of FMPH on interaction between H1R and GHSR1a.

\section{H1R antagonism of olanzapine-activated hypothalamic GHSR1a downstream signaling molecules in NPY-GFP cells}

It is known that GHSR1a activates AMPK and subsequent uncoupling protein-2 UCP2 activity increment, which is required to support the signal transduction system and ensure efficient expression of transcription factors (FOXO1 and p-CREB ) of NPY [26; 27; 28]. To understand the effects of H1R antagonism of olanzapine on the downstream signaling molecules of GHSR1a, the expression of pAMPK, UCP2, FOXO1, and p-CREB was examined in the hypothalamic NPYGFP cells treated with olanzapine $(25 \mu \mathrm{M})$ with or without addition of FMPH $(5 \mu \mathrm{M})$ using Western blots. The levels of pAMPK, FOXO1 and pCREB were significantly increased by olanzapine compared with controls $(\mathrm{p}<0.05$, Fig $5 \mathrm{~A}, \mathrm{C}$ and D). The UCP-2 concentration had a trend of increase in olanzapine group compared with control group, but did not reach statistical significance (Fig 5B). FMPH significantly reversed the olanzapine-induced increment of GHSR1a downstream signaling molecules $(\mathrm{p}<0.05$, Fig 5 A-D). The results suggested that H1R antagonist of olanzapine might activate GHSR1a and its downstream signaling pathway through preventing the heterodimer formation between H1R and GHSR1a, then leading to upregulation of transcription factors for NPY expression. 


\section{Olanzapine analogue (E-Olan) with lower affinity to H1R presented negligible enhancement of pCREB within the nucleus of NPY neurons.}

Previously, we have developed an olanzapine analogue (E-Olan) with reduced H1R binding affinity [29], which does not increase body weight and food intake in rats [30]. Here, we examined the E-Olan on the NPY transcription factor pCREB in hypothalamic NPY neurons using immunocytochemistry. E-Olan did not increased pCREB concentrations in the nucleus of NPY neurons compared with olanzapine dosing $(\mathrm{p}<0.01)$ (Fig 5A and 5B). FMPH significantly decreased the pCREB compared with the control group and blocked olanzapine increasing pCREB suggesting olanzapine's H1R antagonist effect is important for activation of pCREB. Since many of centrally-NPY release neurons are GABAergic $[31 ; 32 ; 33]$, we performed double labeling GAD65 with pCREB in NPY neurons (Fig 5A). We found that pCREB and GAD65 co-localized in the hypothalamic NPY neurons, suggesting the olanzapine activated pCREB in GABA interneurons in hypothalamus.

\section{H1R agonist FMPH suppressed olanzapine induced excessive food intake.}

Olanzapine treated rats significantly increased the food intake for the first 4 hours and 16 hours compared with control rats after icv injection of saline $(\mathrm{p}<0.001$, Fig 7$)$. Both FMPH icv injection at 100 and $200 \mathrm{nmol}$ inhibited the olanzapine-elevated food intake for 4 and 16 hours compared with olanzapine group with vehicle icv injection $(p<0.05$, Fig 7$)$. FMPH icv injection did not significantly alter food intake of rats without olanzapine treatment. These results confirmed that the H1R antagonism of olanzapine caused increased food intake. 


\section{Discussion}

In this study, we found that olanzapine: 1) increased NPY concentrations in hypothalamic neurons, which were prevented by H1R agonist (FMPH), 2) did not increase hypothalamic NPY of H1R knockout mice; 3 ) inhibited the interaction of H1R to GHSR 1a, 4) increased the signaling molecule expression downstream of GHSR1a via antagonising H1R. Furthermore, the olanzapine analogue (E-Olan) with reduced H1R binding affinity had negligible effect on NPY transcription factor pCREB of hypothalamic NPY neurons. Finally olanzapine induced excessive food intake was inhibited by H1R agonist FMPH in rats. These findings suggest that the H1R antagonist property of olanzapine is important to reduce the interaction of H1R to GHSR1a, which contributed to an increased NPY via activation of GHSR1a downstream signaling pAMPK-UCP2-FOXO1-pCREB in hypothalamic neurons. This is an important mechanism of olanzapine induced hyperphagia.

Accumulating data have demonstrated that the incidence of obesity and metabolic disorders in schizophrenic patients is associated with antipsychotic agents with high affinity for the H1R including olanzapine, clozapine and risperidone $[34 ; 35 ; 36]$, suggesting the role of H1R in antipsychotic drug-induced obesity. Our previous studies have found that olanzapine increased body weight gain and hyperphagia via increasing orexigenic NPY level in the hypothalamus [23; 37]. This study showed that olanzapine could directly regulate NPY expression presenting an inverted U-shape dose-response effect. H1R antagonist PYR increased NPY level similar to with olanzapine, while H1R agonist FMPH inhibited NPY level in the hypothalamic neurons. Furthermore, olanzapine fail to increase NPY in the hypothalamic neurons of H1R knockout mice. 
Therefore, it is the H1R antagonistic property of olanzapine that caused an increase of orexigenic NPY in the hypothalamic neurons.

It is known that GHSR1a has an unusually high level of constitutive activity, i.e.: it can be active in the absence of a natural ligand ghrelin [38]. GHSR1a is expressed in the NPY neurons and regulates the NPY expression. Recent data indicate that GHSR1a creates a dimer with several Gprotein coupled receptors involved in appetite control and reward [39; 40]. The present work represents the first evidence of direct interaction of H1R and GHSR1a in hypothalamic neurons. We found that H1R co-localises with GHSR1a in the hypothalamic NPY neurons, forming a heterodimer detected by confocal FRET. The heterodimerization may serve as an allosteric mechanism to modulate specific GHS-R1a signaling pathways and receptor functionalities, independently of ghrelin binding to GHSR1a. Furthermore, the interaction between H1R to GHSR1a was increased by H1R agonist (FMPH) but reduced by H1R antagonist (PYR) in NPY neurons treated with olanzapine. Olanzapine inhibited FMPH-induced interaction between H1R to GHSR1a in NPY neurons, suggesting that olanzapine's H1R antagonism inhibits the interaction of H1R to GHSR1a in the hypothalamus.

GHSR1a increases NPY, stimulates appetite, and weight gain via the AMPK- FOXO1-pCREB signalling pathway $[12 ; 27 ; 41 ; 42 ; 43]$. We previously found that olanzapine increased GHSR 1a signalling and NPY in the rat hypothalamus [23]. Here, we found that FMPH prevent olanzapine induced upregulation of GHSR1a signalling AMPK- FOXO1-pCREB in hypothalamic NPY neurons. The pCREB is a transcription factor of NPY. We showed that olanzapine activated pCREB in hypothalamic NPY neurons. FMPH blocked elevation of pCREB induced by olanzapine, which suggested that olanzapine's H1R antagonist effect is important for activation of pCREB as 
a transcription factor for NPY. We have further demonstrated that these NPY neurons express GABA, indicating they are hypothalamic interneurons. Our previous study shows when H1R antagonist property is reduced, E-Olan no longer induces weight gain in rats [29]. The present study found that olanzapine analogue, E-Olan, with lower affinity to H1R did not activate the NPY transcription factor pCREB in the hypothalamic neurons.

In summary, we have demonstrated that H1R creates a dimer with GHSR1a in the hypothalamic NPY neurons. The mechanism is that H1R antagonism of olanzapine disrupted the inhibition effect of H1R on GHSR1a; therefore increasing AMPK-FOXO1-pCREB signalling by which it increased NPY concentrations in the hypothalamic neurons leading positive energy balance regulation. The new compound we have developed by removing H1R antagonism of olanzapine may provide a better treatment for schizophrenia due to a reduced side-effect of metabolic disorder; however, further study is required prior to human clinical trial.

\section{Method}

Reagents and drugs: Antibodies used in this study were as follows: For immunocytochemistry first antibodies: goat GHSR1a, mouse H1R, rabbit NPY (all from Santa Cruz Biotechnologies; dilution factor 1:250), Mouse GAD-65 (Chemicon 1:250); Secondary antibodies: Donkey antigoat-Alexa Fluor 488, Donkey anti-rabbit-Alexa Fluor 488, Donkey-anti-mouse-Alexa Fluor 555, Donkey-anti-goat-Alexa Fluor 647, Donkey-anti-rabbit-Alexa Fluor 568 all from pCREB (Santa Cruz Biotechnologies; dilution factor 1:250 IF, 1:1000 WB), pAMPK, AMPK, FOXO1 (Cell Signaling Technology 1:1000), UCP2 (Santa Cruz Biotechnologies; dilution factor 1:1000). Secondary antibodies for WB were anti-rabbit (for AMPK, pAMPK, FOXO1 and pCREB) or anti- 
goat (for UCP-2) IgG conjugated with horseradish peroxidase (Santa Cruz Biotechnologies, USA; dilution factor 1:5000). 2-(3-trifluoromethylphenyl) histamine (FMPH), Pyrilamine (PYR) and Olanzapine were obtained from Sigma-Aldrich (Sigma, NSW, Australia).

Animals and Neuronal cultures: Mouse hypothalamic cell line (mHypoA-59, NPY-GFP neurons,

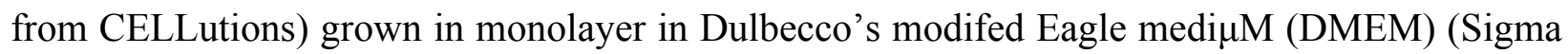
D5796, Castle Hill, NSW) with 10\% fetal bovine ser $\mu$ M (FBS) (SAFC Biosciences Inc, Lenexa, KS) and $1 \%$ penicillin/streptomycin. They were maintained at $37{ }^{\circ} \mathrm{C}$ with $5 \% \mathrm{CO}$. Primary hypothalamus neurons were from hypothalamic tissues of postnatal day 0 (PN0) of wild type-mice or CRISPR/Cas9-mediated gene knockout mice. H1R genotype was identified by Gene Sequencing. Primary hypothalamic cell cultures were prepared based on previous literature [44]. The culture condition was adapted according to Johns Hopkins online protocol [45] with the culture medium generally consisting of a NeuroBasal medium with an additive B27 and extra glucose and glutamine. Mouse hypothalamic cell line (mHypoA-59, NPY-GFP neurons, from CELLutions) grown in monolayer in Dulbecco's modified Eagle medium (DMEM) (Sigma D5796, Castle Hill, NSW) with 10\% foetal bovine serum (FBS) (SAFC Biosciences Inc, Lenexa, KS) and $1 \%$ penicillin/streptomycin. Cells for NPY production assay by flextation were cultured in $96-$ well microtiter plates; Cells for Immunofluorescence were plated into polyD-lysine (P6407, SigmaAldrich) coated coverslips in a $24-w e l l$ plate and maintained at $37^{\circ} \mathrm{C}$ with $5 \% \mathrm{CO}$; Cells for Western blot were cultured in 6-well plates. The procedures on animals for primary hypothalamic neurons were approved by the Animal Ethics Committee, University of Wollongong, NSW, Australia, and carried out in accordance with the Australian Code of Practice for the Care and Use of Animals for Scientific Purposes. 
The Detection of NPY Production by flextation: NPY-GFP neurons were seeded at a density of $1.0 \times 10^{5}$ cells $/ \mathrm{mL}$ and maintained for 24 hours cultivation. Olanzapine, PYR and FMPH with concentration gradient (for olanzapine: $1-100 \mu \mathrm{M}$; for FMPH0.5-10 $\mu \mathrm{M}$; for PYR 0.5-50 $\mu \mathrm{M}$ ) was applied to the neuron independently for prolonged 3 hours culture. NPY production was represented by NPY-bound GFP immunoactivity read by the Flexstation 3 multiplate fluorometer (Molecular Devices Corporation, Sunnyvale, CA). Wavelength of detection was set from $488 \mathrm{~nm}$ to $510 \mathrm{~nm}$, cutoff at $495 \mathrm{~nm}$.

Immunofluorescence and image analysis: For immunochemical staining, neurons cultured on coverslips were fixed with 4\% paraformaldehyde in Dulbecco's PBS for $20 \mathrm{~min}$ at room temperature. The samples were further incubated with $100 \%$ methanol for $10 \mathrm{~min}$ in $-20^{\circ} \mathrm{C}$, and blocked with 5\% donkey serum in PBST for 1 hour at room temperature. Then, for the colocalization of H1R and GHSR1a in NPY-GFP neurons, primary antibodies against H1R (rabbit) and GHSR1a (goat) were applied and incubated for $24 \mathrm{~h}$ at $4{ }^{\circ} \mathrm{C}$. Species-specific secondary antibodies conjugated with Alexa Fluor 568 and 647 were applied for further 2 hours incubate at room temperature. The slips were taken out and sealed on slides in dark for confocal examination. Same procedures were conducted to prepare primary hypothalamus neurons, anti-NPY (rabbit) anti-H1R (mouse) anti-GHSR1a (goat) were used as primary antibodies, secondary antibodies conjugated with Alexa Fluor 488, 568 and 647 were used to visualize NPY, H1R and GHSR1a respectively. Primary antibodies to pCREB (rabbit), GAD65 (mouse) and species-specific secondary antibodies conjugated with Alexa Fluor 488 and 555 were used to measure the expression of pCREB in nucleus of NPY neurons. For the test of NPY and H1R in hypothalamus 
arcuate neurons of wildtype mice, as well as of H1R knock-out mice, the antibodies were antiNPY (rabbit) anti-H1R (mouse) and conjugated with Alexa Fluor 488 (NPY) 555 (H1R). The slides were observed under confocal microscope (Leica-TCS-SP5 Advanced System, Leica Microsystem) and obtained images of target receptors individually. The immunoreactivity of pCREB H1R and NPY in related neurons were quantified by Software Image J. (http://rsbweb.nih.gov/ij/download.html) from 20 neurons collected from 3 independent culture wells. Each experiment was repeated 3 times.

Confocal Microscopy FRET and Data Processing: Paraformaldehyde-fixed NPY neurons in 24wells plates coated with coverslips were incubated for $24 \mathrm{~h}$ at $4{ }^{\circ} \mathrm{C}$ with primary antibodies highly specific to H1R and GHSR1a receptors then the specie-specific secondary antibodies conjugated to Alexa Fluor 568 and Alexa Fluor 647 dyes, respectively. Anti-H1R-Alexa Fluor 568 was used as a donor dipole, while anti-GHSR1a-Alexa Fluor 647 was used as acceptor dipole. The images were obtained by Olympus Fluoview FV 1000 laser scanning confocal microscope with 63/1.4 NA objective. The donor was excited with a krypton laser at $561 \mathrm{~nm}$, while the acceptor was excited with an argon laser at $633 \mathrm{~nm}$. The emissions were collected with 620/40nm and 680/30nm LP filter.

Primary hypothalamus neurons were paraformaldehyde-fixed and incubated with primary antibodies to GHSR1a and H1R receptors then dyed by specie-specific secondary antibodies conjugated to Alexa Fluor 488 and 568 individually. Anti-GHSR1a-Alexa Fluor 488 was used as a donor dipole, while anti-H1R-Alexa Fluor 568 was used as acceptor dipole. The donor was excited with a krypton laser at $488 \mathrm{~nm}$, while the acceptor was excited with an argon laser at 561 
$\mathrm{nm}$. The emissions were collected with 530/30 and 620/40nm LP filter. 20 images were acquired for each FRET analysis according to referred algorithm [46; 47]. FRET efficiencies (E) for the sensitized emission were calculated and analyzed using FRET-SE Wizard Software (Leica Microsystems Pty Ltd, NSW Australia). The distance (r) between the donor and the acceptor was calculated by selecting small regions of interest (ROI) using the equation $r=R 0[(1 / E)-1] 1 / 6 . R 0$ is the Förster's distance. The FRET efficiencies directly indicate the distance between donor and acceptor [48].

Western blot: NPY-GFP neurons cultured in 6-wells plates and treated by olanzapine and the combination of olanzapine and FMPH. Cells were harvested using lysis buffer (containing NP40, Protease Inhibitor Cocktail, $1 \mathrm{mM}$ PMSF and $0.5 \mathrm{mM} \beta$-glycerophosphate). The protein concentrations were determined by DC ${ }^{\mathrm{TM}}$ Protein Assay kit (5000121, Bio-Rad, USA) and detected through a SpectraMax Plus384 absorbance microplate reader (Molecular Devices, USA). $10 \mu \mathrm{g}$ proteins of each sample were heated in Laemmli buffer at $95^{\circ} \mathrm{C}$ before loaded to $8 \%$ SDSPAGE gels for fractionation, followed by Electrophoresis under $120 \mathrm{~V}$ for 1.5 hours. Then the proteins were transferred onto Immun-BlotTM PVDF membranes (Bio-Rad, USA). After blocked in 5\% BSA (diluted in TBST), the membranes were incubated with pCREB antibody (Santa Cruz Biotechnologies; dilution factor 1:1000) and pAMPK, AMPK, UCP2 and FOXO1 antibodies (Cell Signalling Technology; dilution factor 1:1000) separately in TBST containing 1\% BSA overnight at $4{ }^{\circ} \mathrm{C}$. Secondary antibodies were anti-rabbit $\operatorname{IgG}$ conjugated with horseradish peroxidase for pAMPK, AMPK, FOXO1 and pCREB (Santa Cruz, CA; dilution factor 1:5000). Secondary antibody against UCP2 was goat IgG conjugated with horseradish peroxidase (Santa Cruz, CA; dilution factor 1:5000). For visualization, ECL detection reagents were used and films were 
exposed on the AGFA CP1000 Tabletop Processor (AGFA Healthcarem Scoresby, VIC). Films were analyzed using Quantity One software connected to a GS-690 Imaging Densitometer (BioRad, Hercules, and CA).

FMPH intervention: To further investigate $\mathrm{H} 1$ antagonism of olanzapine induced food intake, an H1 receptor agonist, FMPH was injected in olanzapine treated rats. Briefly, a 24-gauge guide cannula was implanted with into the lateral ventricle of $\mathrm{SD}$ rat $(1.0 \mathrm{~mm}$ posterior to the bregma, $1.5 \mathrm{~mm}$ lateral to the midline, and $3.5 \mathrm{~mm}$ below the top skull) [49]. After 1 week, the rats were given olanzapine or vehicle for 4 days. A cookie dough pellet (62\% carbohydrate, $22 \%$ protein, $6 \%$ fiber, and $10 \%$ vitamins and minerals) mixed with either olanzapine $(1 \mathrm{mg} / \mathrm{kg})$ or placebo 3 times daily at 8-hour intervals at $7 \mathrm{am}, 3 \mathrm{pm}$, and $11 \mathrm{pm} \mathrm{[50].} \mathrm{On} \mathrm{day} \mathrm{5,} \mathrm{two} \mathrm{dosage} \mathrm{of} \mathrm{H1}$ receptor agonist, FMPH, (100 or $200 \mathrm{nmol})$ or saline as a control were injected into lateral ventricle of rats

(in $5 \mu \mathrm{L}$, at a rate of $5 \mu \mathrm{L} / \mathrm{min}$ ). Thirty minutes later, the rats were given olanzapine or vehicle in cookie dough pellet. We measured the food intake of the rats at 4 and 16 hours after the olanzapine or vehicle were administered.

Statistical analysis: SPSS (version 21; Chicago, IL) was used for statistical analysis. One-way analysis of variance (ANOVA) with post-hoc Tukey's tests was performed for multiple comparisons. Data were expressed as mean $\pm \mathrm{SEM}$, and $\mathrm{p}<0.05$ was considered statistically significant.

\section{Figure Legends}

Fig 1. A: Olanzapine (Ola) stimulated neuropeptide immune-reactivity (NPY-IR) in a dose response manner in hypothalamic NPY-GFP neurons in 3 hours. B: H1R agonist 2-(3- 
trifluoromethylphenyl) histamine dihydrogenmaleate (FMPH) inhibited NPY-IR in 3 hours. C: H1R antagonist pyrilamine (PYR) stimulated NPY-IR in a pattern similar to olanzapine in 3 hours. ${ }^{*} \mathrm{p}<0.05$ vs. nil control; **: $\mathrm{p}<0.01$ vs. nil control.

Fig 2. Fluorescence immune-reactivity of neuropeptide Y (NPY) and histamine 1 receptor (H1R) in cultured hypothalamic neurons. A: The NPY neurons (Green) of the hypothalamic arcuate nucleus express H1R (red) and co-localised (Merge). B: Olanzapine (Ola, 25 $\mu \mathrm{M})$ increases NPY expression in wildtype, but not H1R-KO mice. ${ }^{* *} \mathrm{p}<0.01$ and ${ }^{* * *} \mathrm{p}<0.001$.

Fig 3. Triple immunofluorescence labelling showing co-localisation of NPY, H1R, and GHSR1a in the neuron of hypothalamic arcuate nucleus using primary cell culture (Row A). Row-B shows that GHSR1a and H1R are co-localised (Merge) form heterodimer (FRET, purple). Row-C indicates that GHSR1a does not show FRET without adding H1R antibody (no fluorescence donor). Row-D indicates that H1R does not show FRET without adding GHSR1a antibody (no fluorescence acceptor). The FRET was increased by H1R antagonist FMPH, but decreased by Olan and H1R agonist in soma and dendrites (E and F) of primary hypothalamic neurons. ${ }^{*} \mathrm{p}<0.05$ vs. control $(\mathrm{CON}),{ }^{* *} \mathrm{p}<0.01$ vs. CON and ${ }^{\#} \mathrm{p}<0.01$ vs. FMPH.

Fig 4. Fluorescence immunoreactivity of NPY-GFP neurons express H1R and GHSR1a, which are FRET positive. Row-A shows co-localization of NPY, H1R and GHSR1a (Merge). Row-B shows that FRET is positive (purple) indicating H1R and GHSR1a interaction. C shows that H1R antagonist FMPH increased FRET but olanzapine (OLA) prevented FRET induced by OLA. ${ }^{* *} \mathrm{p}<0.01$ vs. control, ${ }^{\#} \mathrm{p}<0.01$ vs. FMPH. 
Fig 5. The expression of pAMPK $\alpha$-FOXO1-pCREB signaling in response to olanzapine (OLA) and H1R agonist FMPH. OLA increases pAMPK $\alpha$, FOXO1, and pCREB which can be prevented by FMPH in hypothalamic NPY-GFP neurons. ${ }^{*} \mathrm{p}<0.05$ vs CON, $\# \mathrm{p}<0.05$ vs Olan.

Fig 6. The pCREB immunoreactivity in the nucleus of hypothalamic NPY-GFP neurons is regulated by H1R activity. The first row shows that pCREB was stimulated by olanzapine (Ola), but not olanzapine with reduced H1R activity (OlzEt). H1R agonist FMPH reduced pCREB which was reversed by Ola. The second row showed these neurons are GAD65 positive which was not affected by any treatment used here. The third row showed double labelling of pCREB and GAD65. B shows the quantitative data of $A(n=20),{ }^{*} \mathrm{p}<0.05,{ }^{* *} \mathrm{p}<0.01$ vs. control. The NPY transcription factor pCREB is positive in GAD65, suggesting these neurons are hypothalamic interneurons.

Fig 7. H1R agonist FMPH administered into intracerebral ventricle inhibiting olanzapine (OLA)induced excessive food intake for 4 and 16 hours. All data were presented as mean \pm SEM. *: $\mathrm{p}<0.05, * *: \mathrm{p}<0.01, * * *: \mathrm{p}<0.001$ vs. Ola. FMPH1: 100 nmol, FMPH2: 200 nmol FMPH $(\mathrm{n}=5-$ 8 mice per group). 


\section{References:}

[1] M.C.P. Mauri, S.Di Pace, C.Reggiori, A.Cirnigliaro, G.Valli, I.Altamura, A. C., Clinical Pharmacokinetics of Atypical Antipsychotics: An Update. Clin Pharmacokinet (2018).

[2] L.E. Rojo, P.A. Gaspar, H. Silva, L. Risco, P. Arena, K. Cubillos-Robles, and B. Jara, Metabolic syndrome and obesity among users of second generation antipsychotics: A global challenge for modern psychopharmacology. Pharmacological research 101 (2015) 74-85.

[3] R. Mandrioli, M. Protti, and L. Mercolini, Novel Atypical Antipsychotics: Metabolism and Therapeutic Drug Monitoring (TDM). Curr Drug Metab 16 (2015) 141-51.

[4] J.W. Newcomer, Second-generation (atypical) antipsychotics and metabolic effects: a comprehensive literature review. CNS Drugs 19 Suppl 1 (2005) 1-93.

[5] G.E. Nicol, M.D. Yingling, K.S. Flavin, J.A. Schweiger, B.W. Patterson, K.B. Schechtman, and J.W. Newcomer, Metabolic Effects of Antipsychotics on Adiposity and Insulin Sensitivity in Youths: A Randomized Clinical Trial. JAMA Psychiatry 75 (2018) 788796.

[6] P.H. Hou, G.R. Chang, C.P. Chen, Y.L. Lin, I.S. Chao, T.T. Shen, and F.C. Mao, Long-term administration of olanzapine induces adiposity and increases hepatic fatty acid desaturation protein in female C57BL/6J mice. Iran J Basic Med Sci 21 (2018) 495-501.

[7] K. Weston-Green, X.-F. Huang, and C. Deng, Olanzapine treatment and metabolic dysfunction: a dose response study in female Sprague Dawley rats. Behavioural Brain Research 217 (2011) 337-346.

[8] A.T. Raben, V.S. Marshe, A. Chintoh, I. Gorbovskaya, D.J. Muller, and M.K. Hahn, The Complex Relationship between Antipsychotic-Induced Weight Gain and Therapeutic Benefits: A Systematic Review and Implications for Treatment. Front Neurosci 11 (2017) 741.

[9] J. Lian, M. De Santis, M. He, and C. Deng, Risperidone-induced weight gain and reduced locomotor activity in juvenile female rats: The role of histaminergic and NPY pathways. Pharmacol Res 95-96 (2015) 20-6.

[10] J.A. Harrold, T. Dovey, X.J. Cai, J.C. Halford, and J. Pinkney, Autoradiographic analysis of ghrelin receptors in the rat hypothalamus. Brain Res 1196 (2008) 59-64.

[11] Y. Minokoshi, T. Alquier, N. Furukawa, Y.B. Kim, A. Lee, B. Xue, J. Mu, F. Foufelle, P. Ferre, M.J. Birnbaum, B.J. Stuck, and B.B. Kahn, AMP-kinase regulates food intake by responding to hormonal and nutrient signals in the hypothalamus. Nature 428 (2004) 56974.

[12] Z.B. Andrews, Z.W. Liu, N. Walllingford, D.M. Erion, E. Borok, J.M. Friedman, M.H. Tschop, M. Shanabrough, G. Cline, G.I. Shulman, A. Coppola, X.B. Gao, T.L. Horvath, and S. Diano, UCP2 mediates ghrelin's action on NPY/AgRP neurons by lowering free radicals. Nature 454 (2008) 846-51.

[13] M.S. Kim, Y.K. Pak, P.G. Jang, C. Namkoong, Y.S. Choi, J.C. Won, K.S. Kim, S.W. Kim, H.S. Kim, J.Y. Park, Y.B. Kim, and K.U. Lee, Role of hypothalamic Foxol in the regulation of food intake and energy homeostasis. Nat Neurosci 9 (2006) 901-6.

[14] Y. Date, and K. Kangawa, Ghrelin as a starvation signal. Obes Res Clin Pract 6 (2012) e263-346.

[15] C. Canto, Z. Gerhart-Hines, J.N. Feige, M. Lagouge, L. Noriega, J.C. Milne, P.J. Elliott, P. Puigserver, and J. Auwerx, AMPK regulates energy expenditure by modulating NAD+ metabolism and SIRT1 activity. Nature 458 (2009) 1056-60. 
[16] O. Al Massadi, M. López, M. Tschöp, C. Diéguez, and R. Nogueiras, Current Understanding of the Hypothalamic Ghrelin Pathways Inducing Appetite and Adiposity. Trends in Neurosciences 40 (2017) 167-180.

[17] M. He, Q. Zhang, C. Deng, H. Wang, and X.F. Huang, Olanzapine-activated AMPK signaling in the dorsal vagal complex is attenuated by histamine $\mathrm{H} 1$ receptor agonist in female rats. Endocrinology 155 (2014) 4895-904.

[18] M. He, Q. Zhang, C. Deng, H. Wang, J. Lian, and X.F. Huang, Hypothalamic histamine H1 receptor-AMPK signaling time-dependently mediates olanzapine-induced hyperphagia and weight gain in female rats. Psychoneuroendocrinology 42 (2014) 153-64.

[19] A.S.H. Sangwon F. Kim, Adele M. Snowman, Cory Teuscher, and Solomon H. Snyder, Antipsychotic drug-induced weight gain mediated by histamine $\mathrm{H} 1$ receptor-linked activation of hypothalamic AMP-kinase. PNAS 104 (2007) 3456-3459.

[20] M. Han, C. Deng, T.H. Burne, K.A. Newell, and X.F. Huang, Short- and long-term effects of antipsychotic drug treatment on weight gain and $\mathrm{H} 1$ receptor expression. Psychoneuroendocrinology 33 (2008) 569-80.

[21] H. Schellekens, T.G. Dinan, and J.F. Cryan, Taking two to tango: a role for ghrelin receptor heterodimerization in stress and reward. Front Neurosci 7 (2013) 148.

[22] K. Loh, H. Herzog, and Y.C. Shi, Regulation of energy homeostasis by the NPY system. Trends Endocrinol Metab 26 (2015) 125-35.

[23] Q. Zhang, M. He, C. Deng, H. Wang, J. Lian, and X.F. Huang, Hypothalamic ghrelin signalling mediates olanzapine-induced hyperphagia and weight gain in female rats. Int $\mathrm{J}$ Neuropsychopharmacol 17 (2014) 807-18.

[24] M.G. Willesen, P. Kristensen, and J. Romer, Co-localization of growth hormone secretagogue receptor and NPY mRNA in the arcuate nucleus of the rat. Neuroendocrinology 70 (1999) 306-16.

[25] D. Kohno, H. Sone, Y. Minokoshi, and T. Yada, Ghrelin raises [Ca2+]i via AMPK in hypothalamic arcuate nucleus NPY neurons. Biochemical and biophysical research communications 366 (2008) 388-92.

[26] M. Lopez, R. Lage, A.K. Saha, D. Perez-Tilve, M.J. Vazquez, L. Varela, S. SangiaoAlvarellos, S. Tovar, K. Raghay, S. Rodriguez-Cuenca, R.M. Deoliveira, T. Castaneda, R. Datta, J.Z. Dong, M. Culler, M.W. Sleeman, C.V. Alvarez, R. Gallego, C.J. Lelliott, D. Carling, M.H. Tschop, C. Dieguez, and A. Vidal-Puig, Hypothalamic fatty acid metabolism mediates the orexigenic action of ghrelin. Cell metabolism 7 (2008) 389-99.

[27] Z.B. Andrews, Central mechanisms involved in the orexigenic actions of ghrelin. Peptides 32 (2011) 2248-2255.

[28] M. Lopez, Hypothalamic AMPK and energy balance. Eur J Clin Invest 48 (2018) e12996.

[29] S. Jafari, M.E. Bouillon, X.-F. Huang, S.G. Pyne, and F. Fernandez-Enright, Novel olanzapine analogues presenting a reduced $\mathrm{H} 1$ receptor affinity and retained 5HT2A/D2 binding affinity ratio. BMC pharmacology 12 (2012) 8.

[30] M. López, S. Jafari, X.-F. Huang, J.L. Andrews, and F. Fernandez-Enright, In Vivo Pharmacological Evaluations of Novel Olanzapine Analogues in Rats: A Potential New Avenue for the Treatment of Schizophrenia. PloS one 8 (2013) e80979.

[31] E. Polgar, T.C. Sardella, M. Watanabe, and A.J. Todd, Quantitative study of NPYexpressing GABAergic neurons and axons in rat spinal dorsal horn. J Comp Neurol 519 (2011) 1007-23. 
[32] R. Elghaba, N. Vautrelle, and E. Bracci, Mutual Control of Cholinergic and Low-Threshold Spike Interneurons in the Striatum. Front Cell Neurosci 10 (2016) 111.

[33] S. Suyama, and T. Yada, New insight into GABAergic neurons in the hypothalamic feeding regulation. J Physiol Sci (2018).

[34] W.K. Kroeze, S.J. Hufeisen, B.A. Popadak, S.M. Renock, S. Steinberg, P. Ernsberger, K. Jayathilake, H.Y. Meltzer, and B.L. Roth, H1-histamine receptor affinity predicts shortterm weight gain for typical and atypical antipsychotic drugs. Neuropsychopharmacology 28 (2003) 519-26.

[35] M. He, Q. Zhang, C. Deng, T. Jin, X. Song, H. Wang, and X.F. Huang, Time-dependent effects of olanzapine treatment on the expression of histidine decarboxylase, $\mathrm{H} 1$ and $\mathrm{H} 3$ receptor in the rat brain: The roles in olanzapine-induced obesity.

Psychoneuroendocrinology 85 (2017) 190-199.

[36] H. Sato, C. Ito, K. Hiraoka, M. Tashiro, K. Shibuya, Y. Funaki, T. Yoshikawa, R. Iwata, H. Matsuoka, and K. Yanai, Histamine H1 receptor occupancy by the new-generation antipsychotics olanzapine and quetiapine: a positron emission tomography study in healthy volunteers. Psychopharmacology (Berl) 232 (2015) 3497-505.

[37] J. Lian, X.F. Huang, N. Pai, and C. Deng, Ameliorating antipsychotic-induced weight gain by betahistine: Mechanisms and clinical implications. Pharmacol Res 106 (2016) 51-63.

[38] E.J. Lopez Soto, F. Agosti, A. Cabral, E.R. Mustafa, V.M. Damonte, M.A. Gandini, S. Rodriguez, D. Castrogiovanni, R. Felix, M. Perello, and J. Raingo, Constitutive and ghrelin-dependent GHSR1a activation impairs CaV2.1 and CaV2.2 currents in hypothalamic neurons. J Gen Physiol 146 (2015) 205-19.

[39] M. Wellman, and A. Abizaid, Growth Hormone Secretagogue Receptor Dimers: A New Pharmacological Target. eNeuro 2 (2015).

[40] L. Mihalache, A. Gherasim, O. Nita, M.C. Ungureanu, S.S. Padureanu, R.S. Gavril, and L.I. Arhire, Effects of ghrelin in energy balance and body weight homeostasis. Hormones (Athens) 15 (2016) 186-196.

[41] R.C. Rabinovitch, B. Samborska, B. Faubert, E.H. Ma, S.P. Gravel, S. Andrzejewski, T.C. Raissi, A. Pause, J. St-Pierre, and R.G. Jones, AMPK Maintains Cellular Metabolic Homeostasis through Regulation of Mitochondrial Reactive Oxygen Species. Cell reports 21 (2017) 1-9.

[42] D.G. Hardie, AMPK: positive and negative regulation, and its role in whole-body energy homeostasis. Current Opinion in Cell Biology 33 (2015) 1-7.

[43] G.D. Pimentel, E.R. Ropelle, G.Z. Rocha, and J.B.C. Carvalheira, The role of neuronal AMPK as a mediator of nutritional regulation of food intake and energy homeostasis. Metabolism: clinical and experimental 62 (2013) 171-178.

[44] L.G. Hilgenberg, Smith, M. A. Preparation of Dissociated Mouse Cortical Neuron Cultures., Preparation of Dissociated Mouse Cortical Neuron Cultures., 2007.: p.. J. Vis. Exp. 10 (2007) e562.

[45] J.H. Medicine, Dissociated Primary Hypothalamic Neuron Culture Johns Hopkins Medicine. (2015).

[46] Y. Chen, M. Elangovan, and A. Periasamy, FRET Data Analysis : The Algorithm. Molecular Imaging (2005) 126-145.

[47] X.-P. Wang, H.-N. Yu, and T.-S. Chen, Quantitative Fret Measurement Based on Confocal Microscopy Imaging and Partial Acceptor Photobleaching. Journal of Innovative Optical Health Sciences 05 (2012) 1250015. 
[48] D.C. Harris, Applications of Spectrophotometry, Quantitative Chemical Analysis (8th ed.). New York: W. H. Freeman and Co. , 2010, pp. 419-44.

[49] Paxinos G, Watson C. The Rat Brain in Stereotaxic Coordinates. 6th ed Amsterdam, The Netherlands: Elsevier; 2007.

[50] Zhang Q, He M, Deng C, Wang H, Lian J, Huang XF. Hypothalamic ghrelin signalling mediates olanzapine-induced hyperphagia and weight gain in female rats. Int $\mathrm{J}$ Neuropsychopharmacol. 2014;17(5):807-818. 


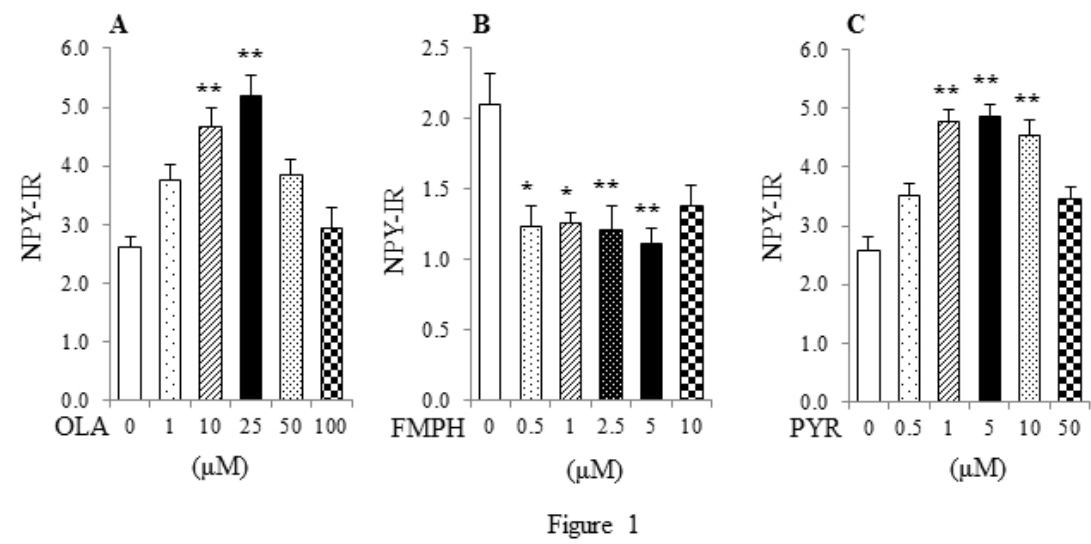




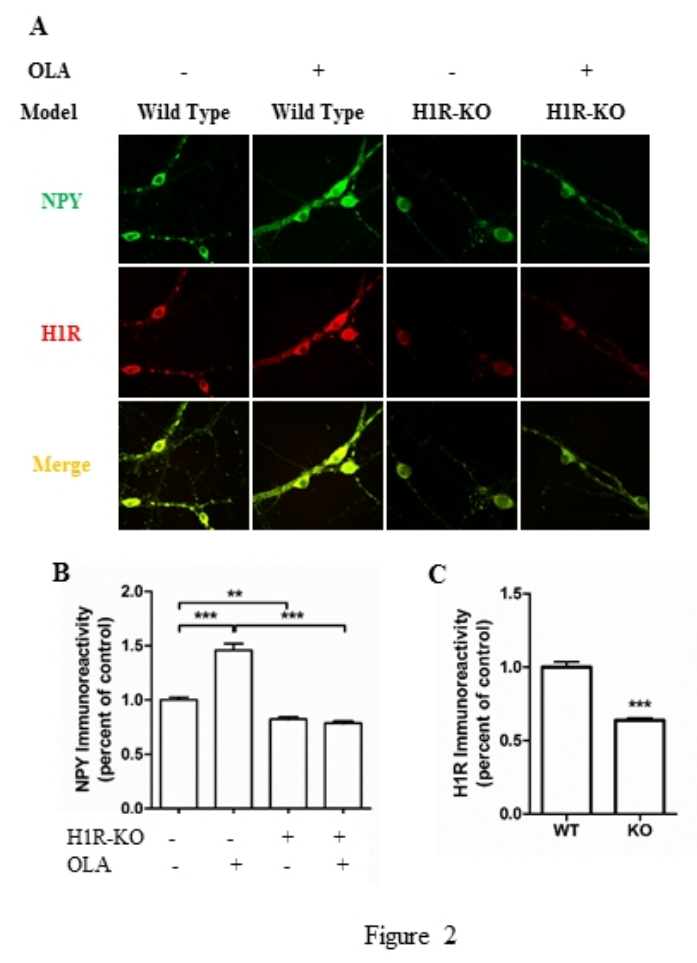




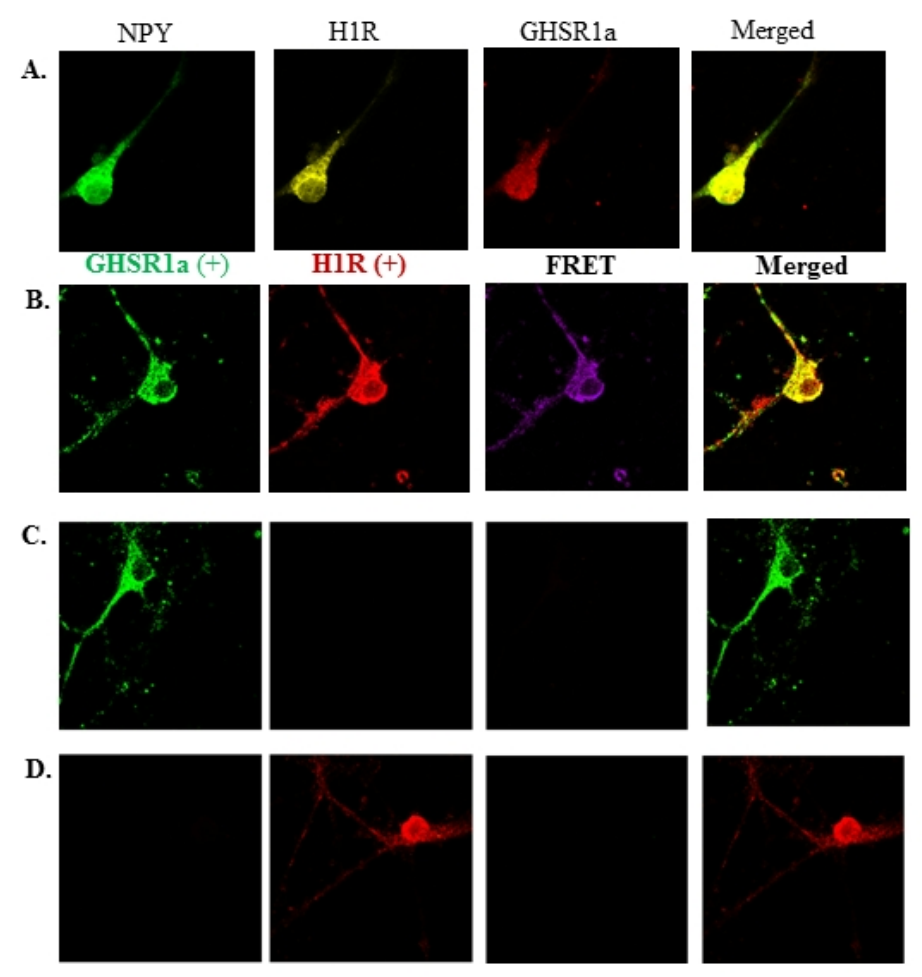

488/510nm

$568 / 603 \mathrm{~nm} \quad$ F. $488 / 603 \mathrm{~nm}$

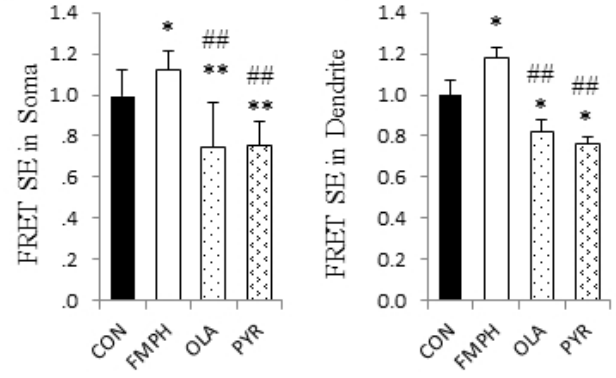

Figure 3 


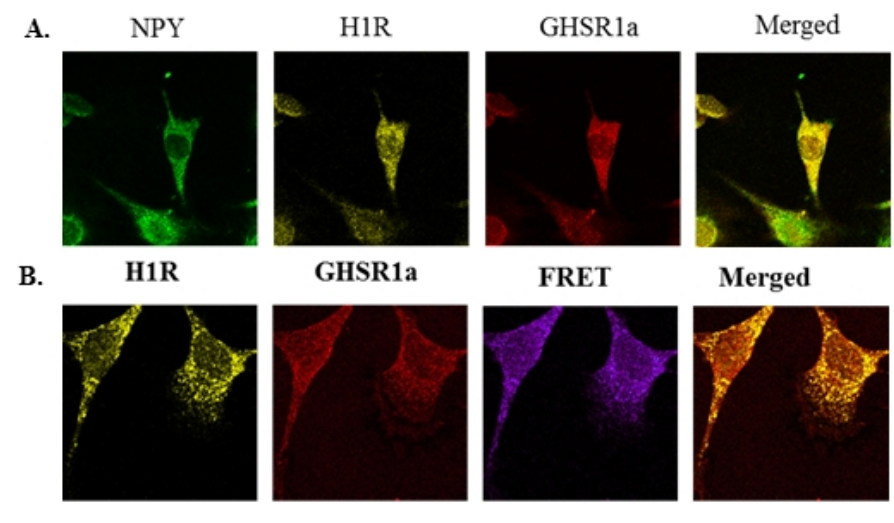

$568 / 603 \mathrm{~nm}$

647/669nm

$568 / 669 n m$

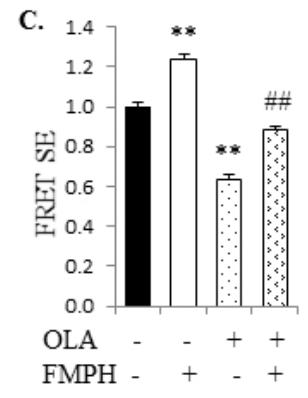

Figure 4 


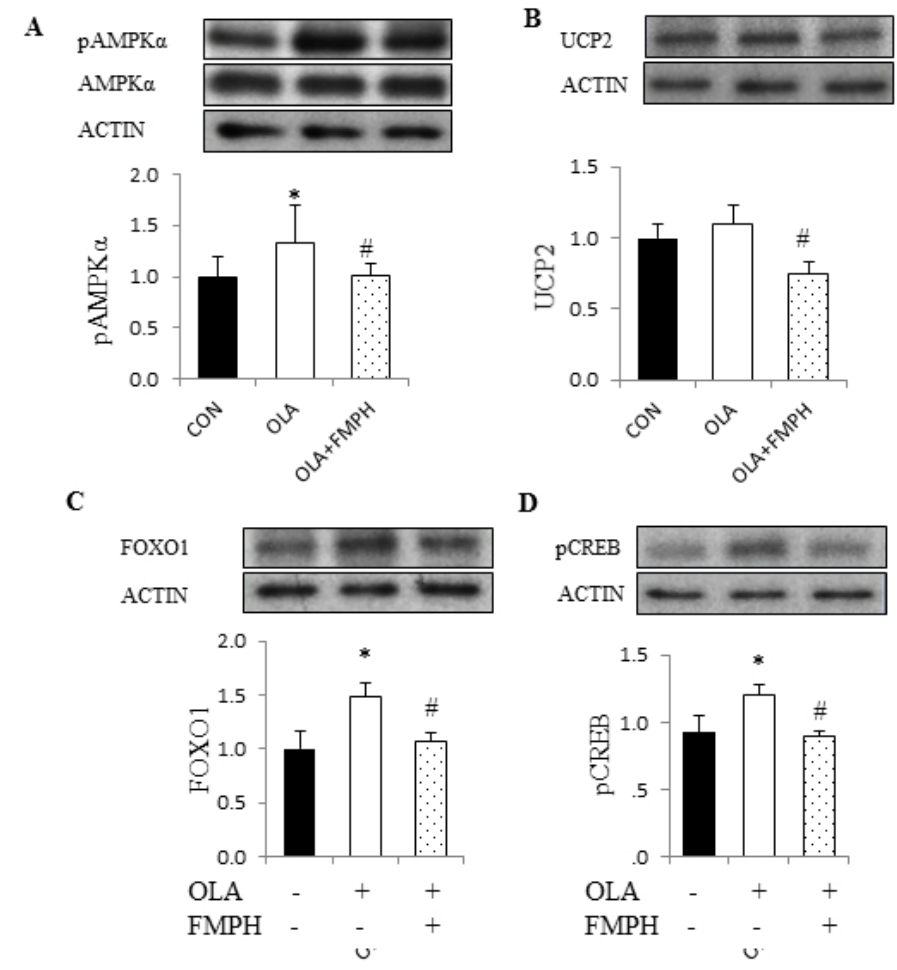

Figure 5 


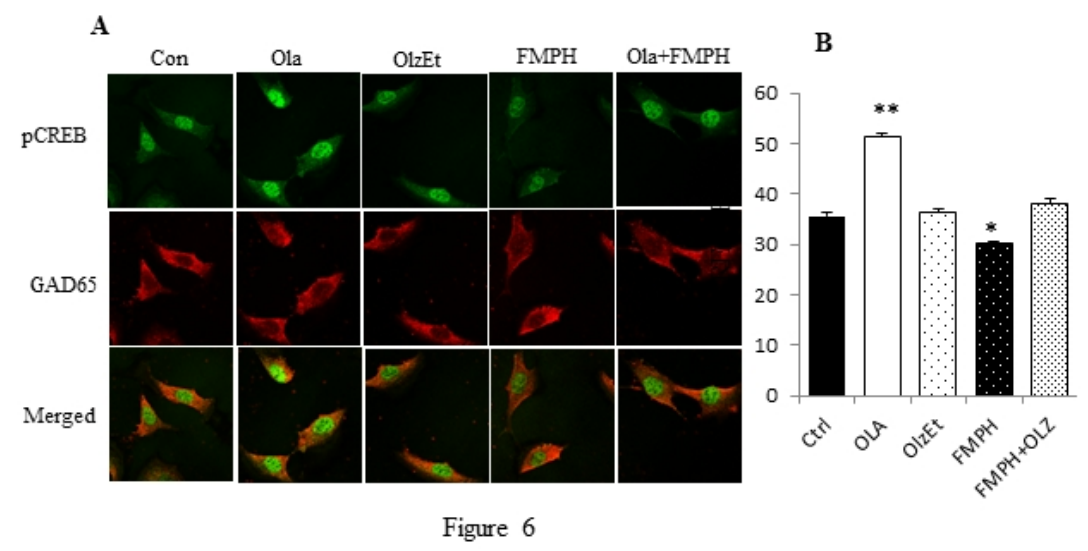




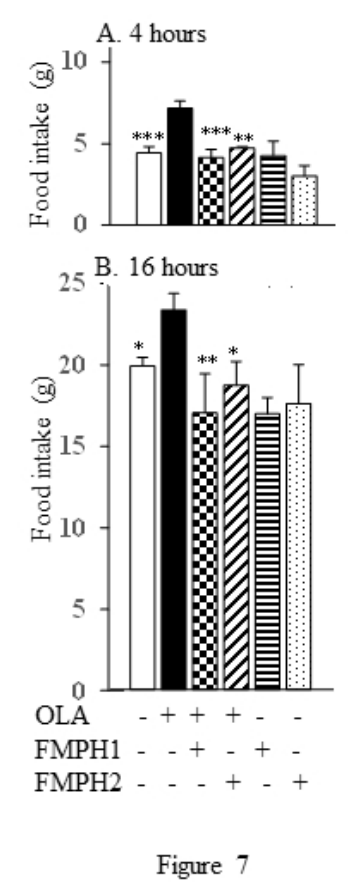

